\title{
Application of Eddy Current Damping Effect to Design a Novel Magnetic Damper
}

\author{
Xiao Denghong ${ }^{*^{1}}$, Zhou Xiaohong ${ }^{1}$, Gao yong ${ }^{1}$, Quan Dongliang ${ }^{1}$, He Tian ${ }^{2}$ \\ 1 Beijing Electro-Mechanical Engineering Institute, Beijing 10074, P. R. China
}

2 School of transportation Science and Engineering, Beihang university, Beijing 10074, P. R. China

xiaodenghong@buaa.edu.cn

Keywords: Eddy current damper, FEM, damping characteristics

Abstract. With advantages of no mechanical contact, vacuum compatibility, oil-free, and high reliability, an eddy current damper (ECD) is designed for use in space. To obtain perfect damping characteristics, a conductor as an outer copper tube and two stationary plates, and an array of axially magnetized ring-shaped permanent magnets separated by iron pole pieces as a mover are used in the proposed ECD. Among the outer conductor tube, a steel tube is used to prevent the electromagnetic leakage. Since the generated eddy currents create are pulsive force that is proportional to the velocity of the conductor, the moving magnet and conductor behave like a viscous damper. An accurate analytical model based on the electromagnetic theory for this type of eddy current damper is proposed, and a finite-element method (FEM) is carried out to predict the magnetic field and current density.

\section{Introduction}

Eddy currents are generated in a conductor in a time-varying magnetic field. They are induced either by the movement of the conductor in the static field or by changing the strength of the magnetic field, initiating motional and transformer electromotive forces, respectively. Since the generated eddy currents create are pulsive force that is proportional to the velocity of the conductor, the moving magnet and conductor behave like a viscous damper. Compared with other types of dampers, such as viscous, viscoelastic, or piezoelectric dampers, the eddy current damper has advantages of no mechanical contact, high reliability, high thermal stability, and vacuum compatibility[1-3]. Although there are those locations that are particularly well suited for eddy current dampers, but perhaps the most promising is in space. Because it did not require any liquid that could leak during operation, had low friction, and provided small variation in damping over a fairly wide range of temperatures. When a device is placed into orbit, the system must function for its entire lifespan without requiring any type of maintenance[4-6]. This can place limitations on the type of damper used, leaving few systems left. The extremely cold temperatures that are present in space actually improve the damping performance of the eddy current damper, due to the decrease in resistivity of the conductor.

This paper proposed a new configuration of eddy current dampers. Figure 2 depicts a schematic configuration of the proposed eddy current damper. The proposed damper consists of a conductor as an outer tube and an array of axially magnetized, ring-shaped permanent magnets separated by iron poles as a mover. Among the outer conductor tube, there is another metal tube of steel material, which is used to prevent the electromagnetic leakage causing damages to components assembled in the spacecraft. This is extremely important for the damper designers. The relative movement of the magnets and the conductor causes the conductor to undergo motional eddy currents. An analytical model for this eddy current damper is derived based on electromagnetic theory, and the eddy current density is computed using finite element analysis FEA. The results show that the new configuration of eddy current damper is effective. 


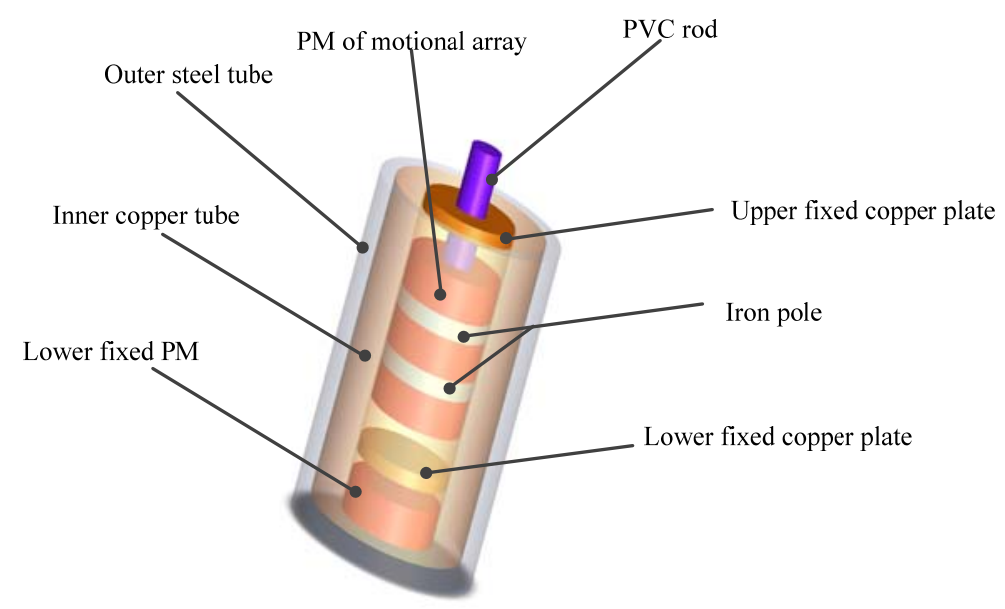

Fig. 1. Schematic view of the proposed ECD.

\section{Description of the prototype and analytical modeling}

The schematic cross-section configuration of the proposed eddy current damper is depicted in Figure 2. Bold arrows indicate the direction of the magnetization for each Permanent magnet (PM). It consists of a ring-shaped conducting aluminum plate and two cylindrical permanent magnets, whose like-poles are in close proximity. The lower permanent magnet and the two eddy current plates are stationary, while the upper magnet array has a reciprocating motion. The copper is chosen as the conductor material because of its high electrical conductively $\left(\sigma=5.8 \times 10^{7} / \Omega \mathrm{m}\right)$. The relative movement of the magnets causes the conducting plate to undergo a time-varying magnetic field such that a transformer eddy current is generated. Since there is a relative movement between magnets and the two conducting plates, a motional eddy current is generated as well.

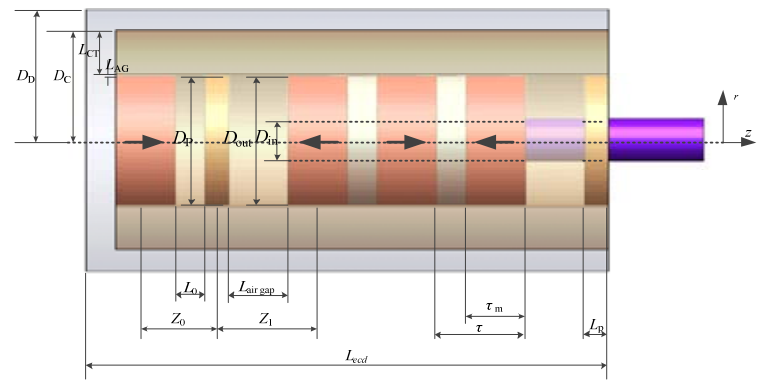

Fig. 2. Configuration of the proposed eddy current damper.

Axially magnetized permanent magnets in the mover result in a higher specific force capability than that of radially magnetized ones. Instead of disc-shaped magnets, annularly shaped magnets supported by a non-ferromagnetic rod are selected; they are fastened on a non-magnetic rod, reducing the effective air gap. Its dimensions are listed in table 1.

Table 1. ECD prototype dimensions

\begin{tabular}{ll}
\hline Item/ symbol & Value/ unit \\
\hline Magnet material & NdFeB alloy $(\mathrm{Br}=1.17 \mathrm{~T})$ \\
Plate and conductor material & copper \\
Magnet diameter (Dout) & $30 \mathrm{~mm}$ \\
Magnet thickness $(\tau \mathrm{m})$ & $10 \mathrm{~mm}$ \\
Magnet inner diameter $(\mathrm{Din})$ & $10 \mathrm{~mm}$ \\
Iron pole thickness $(\tau-\tau \mathrm{m})$ & $5 \mathrm{~mm}$ \\
Plate thickness $(\mathrm{Lp})$ & $4 \mathrm{~mm}$ \\
Air gap (LAG) & $0.5 \mathrm{~mm}$ \\
ECD diameter $(\mathrm{DD})$ & $61 \mathrm{~mm}$ \\
Copper tube outer diameter $(\mathrm{Dc})$ & $51 \mathrm{~mm}$ \\
\hline
\end{tabular}




\section{Magnetic flux density calculation}

There are a number of approaches for calculating the magnetic flux density of a permanent magnet. The simplest approach is the dipole moment model, which is more appropriate for the flux density calculation for long distances, compared with the magnets' diameter. For a permanent magnet with length $\tau \mathrm{m}$ and radius $\mathrm{R}$, the magnetic flux density at a distance $(\mathrm{r}, \mathrm{z})$ from the magnet geometric centre is obtained [7] by computing

$$
\begin{aligned}
& \left.B_{r}(r, z)\right|_{R, \tau_{m}}=\frac{\mu_{0} I}{2 \pi \tau_{m}} \int_{-\tau_{m} / 2}^{\tau_{m} / 2} \frac{\left(z-z^{\prime}\right)}{r\left[(R+r)^{2}+\left(z-z^{\prime}\right)^{2}\right]^{1 / 2}}+ \\
& {\left[-\int_{0}^{\pi / 2} \frac{d \theta}{\sqrt{1-4 R r\left[(R+r)^{2}+\left(z-z^{\prime}\right)^{2}\right]^{-1} \sin ^{2} \theta}}+\cdots+\frac{R^{2}+r^{2}+\left(z-z^{\prime}\right)^{2}}{(R-r)^{2}+\left(z-z^{\prime}\right)^{2}} \times\right.} \\
& \left.\int_{0}^{\pi / 2} \sqrt{1-4 \operatorname{Rr}\left[(R+r)^{2}+\left(z-z^{\prime}\right)^{2}\right]^{-1} \sin ^{2} \theta} d \theta\right] d z^{\prime}
\end{aligned}
$$

where $\mathrm{M}$ is the constant magnetization of the magnet.

For the proposed magnets' configuration in figure 2, the total radial component of the magnetic flux density expelling from each iron pole piece is the sum of the magnetic flux density produced by each adjacent magnets as

$$
B_{r}(r, z)=2\left(\left.B_{r}(r, z)\right|_{D_{\text {out }}, \tau_{m}}-\left.B_{r}(r, z)\right|_{D_{\text {in }}, \tau_{m}}\right)
$$

The distribution of magnetic flux density of eddy current damper is further studied using the FEA method. Both the two-dimensional model and three-dimensional model of the damper are simulated, using ANSYS 13.0, for further analysis of the proposed ECD and to estimate the magnetic flux density [8]. Figure 3 shows the magnetic flux density analysis results of the proposed mover configuration. Magnetic flux density streamlines and the induced magnetic field are plotted in these two figures.

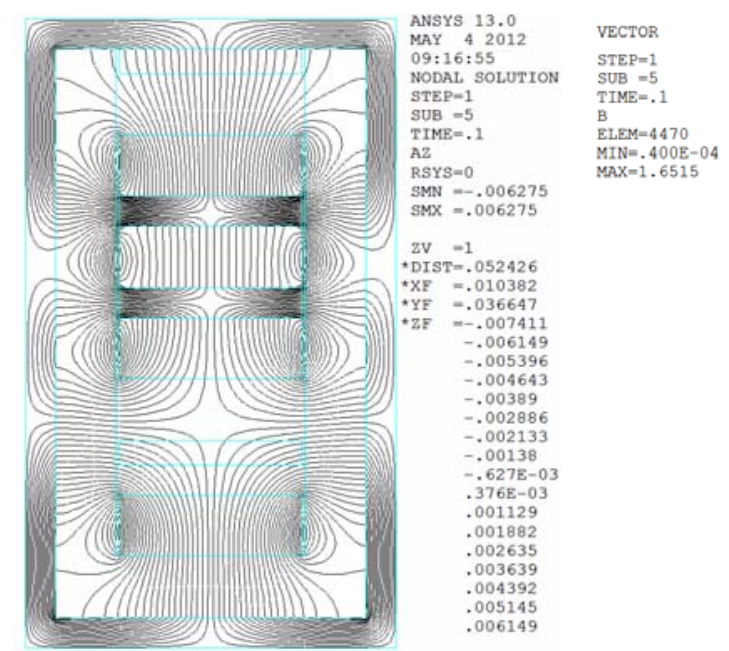

(a)

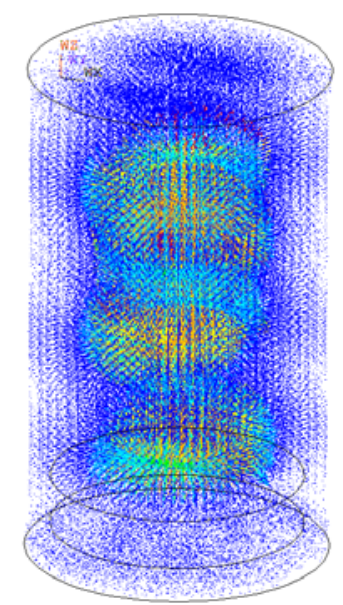

(b)

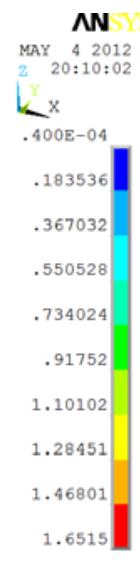

Fig. 3. FE simulation results of the prototype ECD. (a) 2D Flux lines plot of the magnetic flux density; (b) 3D vector plot of the magnetic flux density.

It is observed that the radial magnetic flux density is concentrated and enhanced at the iron pole pieces, causing more eddy current induction such that the ECD is more effective. And the magnetic flux density in areas beyond the ECD is exceedingly weak. That's to say the influence of electromagnetic leakage can be ignored.

Since the 3D finite-element analysis method is much more precise than 2D analysis, the 3D finite-element analysis method is adopted to predict the eddy current density and damping coefficient for conductors moving inside the magnetic field. To add conductor velocity, a 3D transient analysis method is used to simulate a moving conductor under a static magnetic field excitation as moving conductor analysis can be done directly in a 2D static magnetic analysis, but not in 3D in ANSYS. 
And the specific velocity can be assigned to the conductor. The Eddy current distribution of the proposed eddy current damper is depicted in Figure 4.
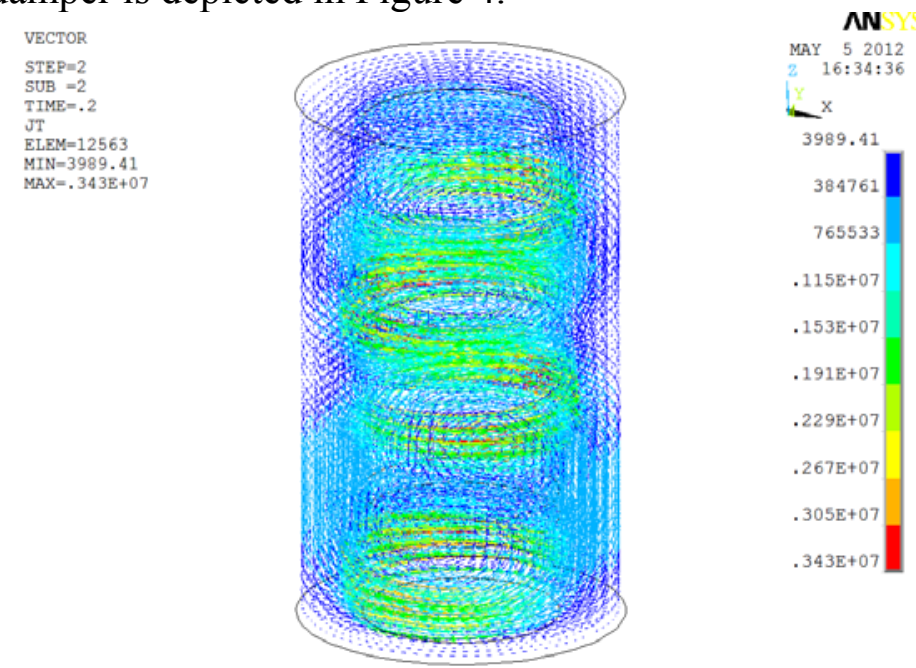

Fig. 4. Eddy current plot of the prototype ECD.

\section{Conclusion}

In this paper, a passive eddy current damper was developed that is capable of effectively suppressing vibrations. The structure of the new passive eddy current damper is straight forward, requiring no external power supply or any other electronic devices. A theoretical model of the proposed system is constructed by using the transformer eddy current contribution for the motional eddy current estimation. The magnetic flux and eddy current damping force are analytically calculated by finite element. The performance of the proposed eddy current damper can be further improved by using high-quality, low-weight permanent magnets, as well as conductors with higher conductivity.

\section{References}

[1] Fung, R-F., Sun, J-H., and Hsu, S-M., 2002, "Vibration Control of the Rotating Flexible-Shaft/Multi-Flexible-Disk System with the Eddy Current Damper," ASME Journal of Vibration and Acoustics, Vol. 124, 519-526.

[2] Matsuzaki, T., Ikeda, T., Nae, A., and Sasaki, T., 2000, "Electromagnetic Forces for a New Vibration Control System: Experimental Verification," Smart Materials and Structures, Vol. 9, No. 2, $127-131$.

[3] Bae, J. S., Kwak, M. K., and Inman, D. J., 2004, "Vibration Suppression of Cantilever Beam Using Eddy Current Damper," Journal of Sound and Vibration, in press.

[4] J.S. Bae, M.K. Kwak, D.J. Inman, Vibration suppression of cantilever beam using eddy current damper, Journal of Sound and Vibration 284 (2005) 805-824.

[5] H. Sodano, J.S. Bae, D.J. Inman, W.K. Belvin, Concept and model of eddy current damper for vibration suppression of a beam, Journal of Sound and Vibration 288 (2005) 1177-1196.

[6] Kienholtz, D. A., Smith, C. A., and Haile, W. B., 1996, “A Magnetically Damped Vibration Isolation System for a Space Shuttle Payload," in Proceeding of the SPIE International Symposium on Smart Structures and Materials, San Diego, CA, Vol. 2720, 272-280.

[7] Babak Ebrahimia, Mir Behrad Khameseea, M. Farid Golnaraghib, Design and modeling of a magnetic shock absorber based on eddy current damping effect, Journal of Sound and Vibration 315 (2008) 875-889. 
[8] He T, Xiao D, Liu X, et al. 920. Design and analysis of a novel eddy current damper based on three-dimensional transient analysis[J]. Journal of Vibroengineering, 2013, 15(1). 